\title{
A Comparative Transcriptomic Approach to Elucidate Common and Divergent Mechanisms Involved in Apricot and Peach Fruit Development and Ripening
}

\author{
G.A. Manganaris, F. Ziliotto, A. Rasori, \\ C. Bonghi and A. Ramina \\ Department of Environmental Agronomy and \\ Crop Science \\ University of Padova \\ viale dell'Università 16 \\ 35020 Legnaro (Padova) \\ Italy
}

\author{
P. Tonutti \\ Scuola Superiore Sant'Anna \\ Piazza Martiri della Libertà, 33 \\ 56127 Pisa \\ Italy
}

Keywords: genomics tools, transcriptomics, oligonucleotide hybridization, maturation, Rosaceae, Prunus armeniaca, Prunus persica

\begin{abstract}
Transcript profiling methods are increasingly used to understand the biological basis of growth and development, and fruit quality in the case of fruits. Such methods provide information for thousands of genes, including those of still unknown function. Furthermore, high-throughput methodologies can be used for comprehensive transcriptome analyses, which may lead to further elucidation of fruit growth and development. Microarray is an attractive genomic tool, since it can be used in a heterologous fashion for gene discovery and characterization in species where few resources are available. In the current study, the progress of apricot (Prunus armeniaca cv. Goldrich) fruit ripening during the last developmental stages was monitored and microarray data that were produced were used for comparative in silico studies with data reported during the transition of peach and nectarine fruits from pre-climacteric to climacteric stage. Transcriptomic studies for both fruit species were carried out using the first available peach microarray ( $\mu$ PEACH 1.0) that contains 4,806 oligonucleotides, each corresponding to a single unigene. Intriguingly, a sharp increase of transcript levels in genes regulating an array of heat shock proteins was detected in apricot fruit, which was not the case during nectarine fruit ripening. In addition, we focused on transcript levels of auxin regulated proteins and their role during the last phases of fruit ripening. Overall, data of the present study offers an initial descriptive picture of transcript profiling of novel key genes and their putative role during the last stages of fruit development is challenged. A future perspective, which will also encompass data validation for genes of interest, is the unravelling of the mechanisms underlying the ripening process in stone-fruits, through the identification of genes differentially expressed during peach and apricot ripening and their correlation with traits of agronomic interest.
\end{abstract}

\section{INTRODUCTION}

Multiple endogenous and exogenous signals influence fruit maturation (on-tree ripening) as well as postharvest ripening and the evolution of pre- and post-harvest quality parameters, respectively. Although physiological processes that regulate the maturation syndrome of fleshy fruits are well documented, few data exist regarding the gene regulation of such processes.

Genomics is the study of genes and their function and is divided into two basic areas: structural genomics (characterization of the physical nature of whole genomes) and functional genomics (characterization of overall patterns of gene expression). The latter is becoming common place in plant science and is mainly based on transcript profiling studies. The aim of this approach is to improve the understanding of complex functional aspects (as growth and development) by detecting changes in expression of genes involved in different cellular processes. Microarray (gene chip) platform is considered as 
a high-throughput tool that allows the simultaneous monitoring of many genes in a single experiment avoiding limitations of traditional molecular techniques; it is an easy way to use sequence information gained from expressed sequence tags (ESTs) and genome sequencing projects and it can be utilized in a heterologous fashion for gene discovery in species with few available resources (Bonghi and Trainotti, 2006). Up to date, a tomato microarray was successfully employed for comparative studies in Solanaceae (tomato, pepper, eggplant) (Moore et al., 2005).

ESTree, the Italian consortium for peach functional genomics (http://www.itb.cnr.it/ ESTree), constructed the first available peach oligonucleotide microarray ( $\mu$ PEACH1.0). The peach platform contains 4,806 oligoprobes ( 70 bases long) each corresponding to a single unigene, as reported in the dedicated database (ESTree consortium, 2005). This unigene collection comes from EST sequences mainly obtained from libraries of ripening fruits, so it is biased towards this physiological process. The application of this platform was used initially to investigate transcriptome changes during transition from preclimacteric to climacteric phase in nectarine (cv. 'Fantasia') fruit (Trainotti et al., 2006). This approach was also used in order to elucidate the involvement of auxin in the ripening of peach (cv. 'Red Haven') and its interplay with ethylene (Trainotti et al., 2007). In addition, microarray tool was applied to elucidate the mode of action of methyl jasmonate (Ziosi et al., 2008) and the effect of 1-methylcyclopropene (1-MCP), an ethylene antagonist in nectarine fruit ripening (Ziliotto et al., 2008).

Considering the high degree of sequence conservation within the Rosaceae family and, in particular, among the Prunus species, the first available peach microarray $(\mu \mathrm{PEACH} 1.0)$ was recently applied to investigate changes in gene expression during transition of apricot (Prunus armeniaca 'Goldrich') from green-immature stage to fullyripe stage (Manganaris et al., 2008). Results indicated that the peach oligo-probe microarray can be successfully applied in a heterologous fashion. The aim of this work was to study transcript profiling of genes during the last stages of on-tree apricot fruit ripening and try to correlate such data with data referring to the transition of peach and nectarine fruits from pre-climacteric to climacteric stage.

\section{MATERIALS AND METHODS}

Fruit material ('Goldrich') was collected at two maturity stages: (1) mature fruit $\left(\mathrm{S}_{\mathrm{m}}\right.$, firmness values $\left.\sim 4 \mathrm{KgF}\right)$, and (2) fully-ripe fruit $\left(\mathrm{S}_{\mathrm{r}}\right.$, firmness values $\left.\sim 1 \mathrm{KgF}\right)$. The RNA was isolated according to Bonghi et al. (1998). In order to remove contaminant DNA from the RNA samples, the nucleic-acid extract was treated with DNase, according to the manufacturer's instructions. The concentration of RNA was quantified by measuring the absorbance at $260 \mathrm{~nm}$ and its integrity was checked on agarose gels. Total RNA $(20 \mu \mathrm{g})$ of each maturity stage was converted into target cDNA by reverse transcription using the SuperScript ${ }^{\mathrm{TM}}$ Indirect cDNA Labeling System (Invitrogen, USA) following manufacturer's instruction. The amino-modified cDNA was coupled to a monoreactive N-hydroxysuccinimide (NHS)-ester fluorescent dye: the green-fluorescent cyanine3 (Cy3) and the red-fluorescent cyanine5 (Cy5) (Amersham Biosciences, UK). A final purification step removed any unincorporated dye. The purity and yield of the labelled cDNA was calculated from the OD values obtained by means of a spectrophotometer (Ultrospec 2100 pro, Amersham Biosciences) using the formulas reported in the SuperScript ${ }^{\mathrm{TM}}$ Indirect cDNA Labeling System instruction manual. The prehybridization and hybridization steps were carried out in Corning ${ }^{\circledR}$ hybridization chambers as analytically described by Trainotti et al. (2007). Three biological replicates were created, repeating three times the same combination of targets one of which was a dye swap and values were subjected to SAM (Significance Analyses for Microarrays) analyses. Genes showing $\log _{2}$ ratio either $>1$ (up-regulated) or $<-1$ (down-regulated) were annotated following the Gene Ontology categories (GO) developed by $\underline{\text { The }}$ Arabidopsis Information $\underline{R}$ esource (TAIR). 


\section{RESULTS AND DISCUSSION}

Among the differentially transcribed genes during transition of apricot fruit from mature to fully ripe stage, an up-regulation of genes encoding heat shock proteins (HSP) was monitored (Fig. 1). These data are in accordance with data reported by Grimplet et al. (2005). Beside their well-documented role as a response to stress conditions, HSP have been involved in pectin depolymerisation (Ramakrishna et al., 2003) and, more recently, were analyzed at a proteomic level during tomato fruit development (Faurobert et al., 2007). In order to detect possible common or divergent mechanisms that govern on-tree fruit ripening in Prunus species, we compared these data with data reported during the transition of peach and nectarine fruit from preclimacteric to climacteric stage (Trainotti et al., 2006, 2007). Intriguingly, results show that the transient increase of transcript levels of hsps appears only in apricot fruit. Whether the examined stages are identical is still questionable; however, this indirect approach may offer some insights about the role of hsps in the ripening syndrome in Rosaceae family.

Members of the AUX/IAA family are highly up-regulated when ripening proceeds and their importance has been highlighted during the onset of peach (cv. Red Haven) fruit ripening (Trainotti et al., 2007). In the case of apricot fruit AUX/IAA appears to remain unaffected offering a field for further research (Fig. 2). However, it is questionable whether the transition of apricot fruit from mature to fully ripe stage is similar to the transition of peach and nectarine fruit from preclimacteric to climacteric stage or the fully ripe stage corresponds to a post-climacteric phase. For certain genes encoding AUX/IAA proteins (ctgs 358, 1741), an increase for the comparison of climacteric to preclimacteric stage has been monitored, while similar expression was the referred level when preclimacteric and postclimacteric stages were compared (Trainotti et al., 2007).

\section{CONCLUSIONS}

The peach gene chip ( $\mu$ PEACH 1.0) can be successfully applied to closely related species as apricot, where fewer information and/or genomics tools are available. Further studies should focus on: i) correlating genes with characteristics of agronomic or physiological interest; ii) the comparison with peach for common and divergent mechanisms during fruit growth, ripening and storage; and iii) the selection and analysis of new candidate genes (e.g auxin regulated, hsp) for a better physiological characterization of apricot fruit development.

\section{ACKNOWLEDGEMENTS}

G.A. Manganaris is a recipient of an E.U. Marie Curie individual fellowship (Grant MEIF-CT-2006-038997). Apricot fruit material was kindly provided by prof. D. Bassi (University of Milan).

\section{Literature Cited}

Bonghi, C., Ferrarese, L., Ruperti, B., Tonutti, P. and Ramina, A. 1998. Endo-beta-1,4glucanases are involved in peach fruit growth and ripening, and regulated by ethylene. Physiologia Plant.102:346-352.

Bonghi, C. and Trainotti, L. 2006. Genomic tools for a better understanding of the fruit ripening process. Stewart Postharvest Review 2:1-10.

ESTreeConsortium. 2005. Development of an oligo-based microarray ( $\mu \mathrm{PEACH} \mathrm{1.0)} \mathrm{for}$ genomics studies in peach fruit. Acta Hort. 682:263-268.

Faurobert, M., Mihr, C., Bertin, N., Pawlowski, T., Negroni, L., Sommerer, N. and Causse, M. 2007. Major proteome variations associated with cherry tomato pericarp development and ripening. Plant Physiol. 143:1327-1346.

Grimplet, J., Romieu, C., Audergon, J-M., Marty, I., Albagnaca, G., Lambert, P., Bouchet, J-P. and Terriera, N. 2005. Transcriptomic study of apricot fruit (Prunus armeniaca) ripening among 13006 expressed sequence tags. Physiologia Plant. 125:281-292.

Manganaris, G.A., Ziliotto, F., Bonghi, C., Banfi, R., Geuna, F., Tonutti, P. and Ramina, A. 2008. Gene expression analyses during ripening of apricot (Prunus armeniaca) 
fruit, using a peach microarray. Acta Hortic. (in press).

Moore, S., Payton, P., Wright, M., Tanksley, S. and Giovannoni, J. 2005. Utilization of tomato microarrays for comparative gene expression analysis in the Solanaceae. J. Exp. Bot. 56:2885-2895.

Ramakrishna, W., Deng, Z.P., Ding, C.K., Handa, A.K. and Ozminkowski, R.H. 2003. A novel small heat shock protein gene, vis1, contributes to pectin depolymerization and juice viscosity in tomato fruit. Plant Physiol. 131:725-735.

Trainotti, L., Bonghi, C., Ziliotto, F., Zanin, D., Rasori, A., Casadoro, G., Ramina, A. and Tonutti, P. 2006. The use of microarray $\mu \mathrm{PEACH1.0} \mathrm{to} \mathrm{investigate} \mathrm{transcriptome}$ changes during transition from pre-climacteric to climacteric phase in peach fruit. Plant Sci. 170:606-613.

Trainotti, L., Tadiello, A. and Casadoro, G. 2007. The involvement of auxin in the ripening of climacteric fruits comes of age: The hormone plays a role of its own and has an intense interplay with ethylene in ripening peaches. J. Exp. Bot. 58:3299-3308.

Ziliotto, F., Begheldo, M., Rasori, A., Bonghi, C. and Tonutti, P. 2008. Transcriptome profiling of ripening nectarine (Prunus persica L. Batsch) fruit treated with 1-MCP. J. Exp. Bot. 59:2781-2791.

Ziosi, V., Bonghi, C., Bregoli, A.M., Trainotti, L., Biondi, S., Sutthiwal, S., Kondo, S. and Costa, G. 2008. Torrigiani, P. Jasmonate-induced transcriptional changes suggest a negative interference with the ripening syndrome in peach fruit J. Exp. Bot. 59:563573 . 


\section{Figures}

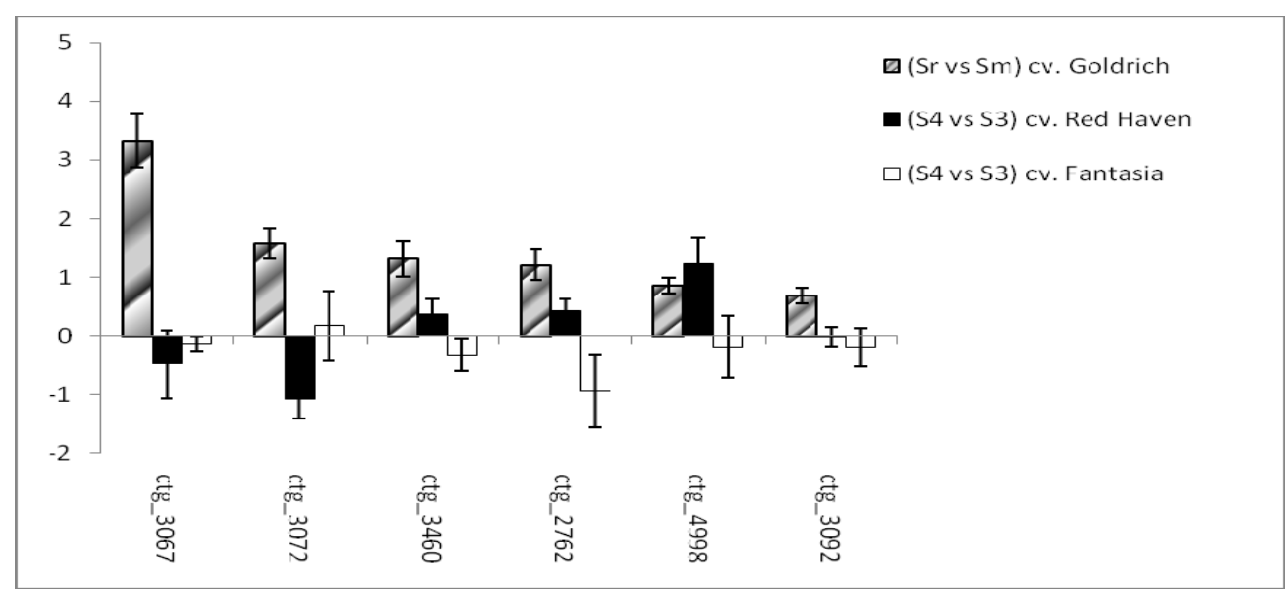

Fig. 1. Modulation of gene expression encoding heat shock proteins, measured by means of the $\mu \mathrm{PEACH} 1.0$ microarray, during the transition of 'Goldrich' apricot fruit from 'mature' to 'ripe' stage. For comparative studies, gene expression profile of these genes during transition of nectarine ('Fantasia') and peach ('Red Haven') fruit from preclimacteric to climacteric stage are presented. (Data adapted from Trainotti et al., 2006, 2007). Bars correspond to \pm SD of the means. ctg_3067: small hsp-soybean, ctg_3072: hsp (Daucus carota), ctg_3460: hsp 70 (Cucumis sativus), ctg 2762: putative hsp 81-2 (HSP 81-2) (Arabidopsis thaliana), ctg_4998: putative hsp (Arabidopsis thaliana), ctg_3092: hsp like protein [imported] (Arabidopsis thaliana).

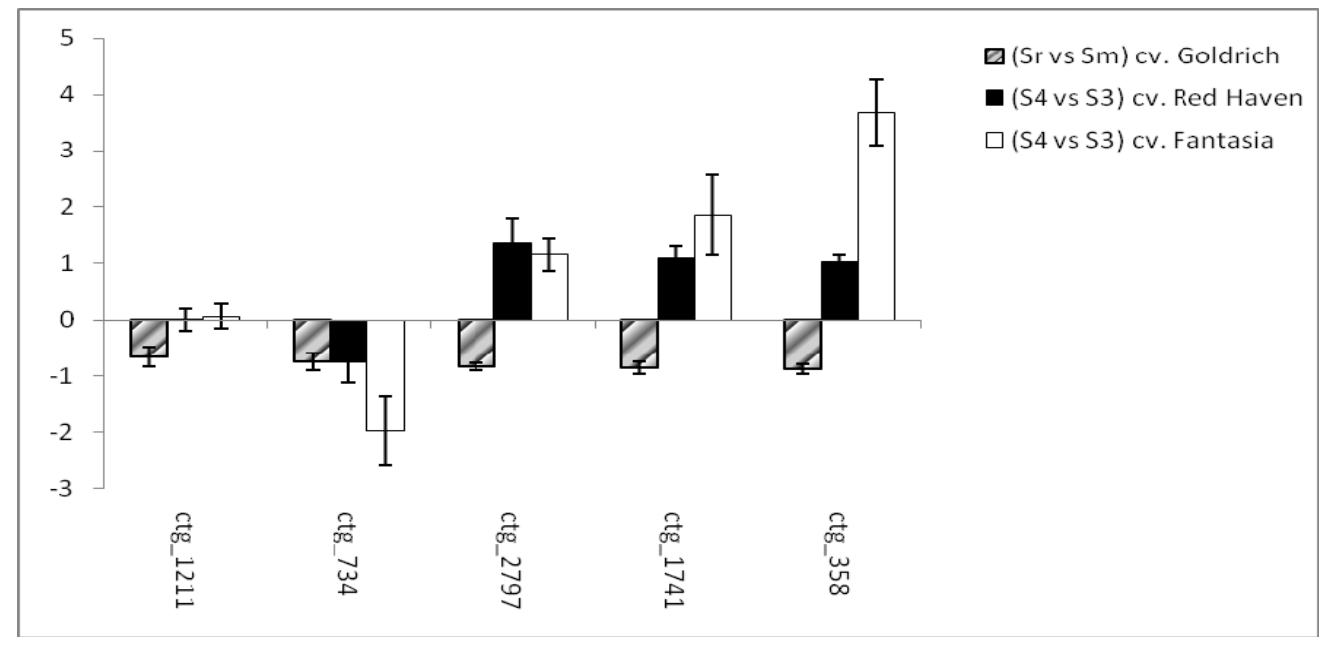

Fig. 2. Modulation of gene expression encoding AUX/IAA proteins, measured by means of the $\mu \mathrm{PEACH} 1.0$ microarray, during the transition of 'Goldrich' apricot fruit from 'mature' to 'ripe' stage. For comparative studies, gene expression profile of these genes during transition of nectarine ('Fantasia') and peach ('Red Haven') fruit from preclimacteric to climacteric stage are presented. (Data adapted from Trainotti et al., 2006, 2007). Bars correspond to \pm SD of the means. ctg_1211: auxin-regulated protein-like protein ctg_734: auxin-repressed protein like-protein ctg_2797: auxin efflux carrier protein family ctg_1741: Aux/IAA protein ctg_358: Aux/IAA protein. 
